RYUTHP-97/1, May 1997

\title{
Dynamical Reduction of Discrete Systems Based on the Renormalization Group Method
}

\author{
T. Kunihiro and J. Matsukidaira \\ Faculty of Science and Technology, Ryukoku University, Seta, Ohtsu, 520-21, Japan
}

(July 3, 2018)

\begin{abstract}
The renormalization group ( $R G$ ) method is extended for global asymptotic analysis of discrete systems. We show that the RG equation in the discretized form leads to difference equations corresponding to the Stuart-Landau or Ginzburg-Landau equations. We propose a discretization scheme which leads to a faithful discretization of the reduced dynamics of the original differential equations.
\end{abstract}

PACS numbers: 11.10.Hi, 64.60.Ak, 03.20.+i, 47.20.Ky, 52.35.Mw, 02.30.mv, 05.45.+b, 63.90.+t 
It is a fundamental problem in physics, especially, in statistical physics since Boltzmann, to reduce many degrees of freedom of a dynamical system to fewer degrees of freedom preserving the essential nature of the system. [1]. The reduced dynamics is often described by a few collective variables which represent slow and long-wave length motion of the system. There are several methods for the dynamical reduction such as the multiple-scale methods (including the reductive perturbation method [2, 3]), the average methods (including Whitham method [四]), the method of normal forms [5] and so on. We are usually interested in asymptotic behavior of the system after a long time. Thus the problem is to obtain a dynamical reduction in asymptotic and global domains.

Recently it has been recognized and emphasized by Chen, Goldenfeld and Oono [7] that the renormalization group equation first developed in quantum field theory [6] is a powerful tool for global and asymptotic analysis: They applied the RG equation à la Gell-Mann-Low to ordinary and partial differential equations and showed that the RG equation nicely gives a reduction of the dynamics describing slow and long-wave length motions of the system; the reduced dynamics is described by so called the amplitude equations. Afterwards the reason of the powerfulness of the RG equations was accounted for in the context of classical theory of envelopes [8]. More recently, it has been shown that the RG method can also naturally leads to the phase equations as well as the amplitude equations [9].

The purpose of this paper is twofold: One is to show that the RG method can be extended to discrete systems and leads to dynamical reduction of discrete dynamical systems (maps). A discrete system is described by a difference equation. We notice that the extension is highly non-trivial because the applicability of the RG method for differential equations is essentially relied on the local nature of differentiation [8]. Another purpose is to propose a discretization scheme for differential equations: It is needless to say that different discretization schemes of non-linear differential equations leads to different dynamical systems. We shall show that such a discretization-scheme dependence also exists for the reducibility of the dynamics and give a good discretization scheme based on the notion of the reducibility of the dynamics.

Now let us take the following discrete system as a typical example of nonlinear discrete 
systems,

$$
x_{n+2}-2 \cos \theta x_{n+1}+x_{n}=\epsilon f\left(x_{n}, x_{n+1}\right),
$$

where $\theta$ is a constant and the function $f\left(x_{n}, x_{n+1}\right)$ contains nonlinear terms. We remark that the equation can be converted to a vector equation, $\mathbf{X}_{n}=\mathbf{F}\left(\mathbf{X}_{n}\right)$ with $\mathbf{X}_{n}={ }^{t}\left(x_{n}, y_{n} \equiv x_{n+1}\right)$ and $\mathbf{F}\left(\mathbf{X}_{n}\right)={ }^{t}\left(y_{n}, 2 \cos \theta y_{n}-x_{n}+\epsilon f\left(x_{n}, y_{n}\right)\right)$. We have taken an example of second-order equation here, but the following discussion is applicable for higher-order equations. We notice that the unperturbed equation $(\epsilon=0)$ has a neutrally stable solution $\exp ( \pm i \theta)$. [11] Assuming that $\epsilon$ is small, we shall apply the perturbation theory. To make the discussion definite, let us take a concrete form for $f\left(x_{n}, x_{n+1}\right)$ as; $f\left(x_{n}, x_{n+1}\right)=a_{1} x_{n}+a_{2} x_{n}^{2}+a_{3} x_{n}^{3}$. If we put $a_{2}=0$, the resultant equation may be considered as a discrete version of the damped Duffin equation.

Expanding $x_{n}$ as $x_{n}=x_{n}^{(0)}+\epsilon x_{n}^{(1)}+\epsilon^{2} x_{n}^{(2)}+\cdots$, let us try to find a solution which is valid around $n=n_{0}$ where $n_{0}$ is arbitrary: $x_{n}^{(i)}(i=0,1, \ldots)$ satisfies $\hat{L} x_{n}^{(0)}=0, \hat{L} x_{n}^{(1)}=$ $a_{1} x_{n}^{(0)}+a_{2} x_{n}^{(0)^{2}}+a_{3} x_{n}^{(0)^{3}}$, and so on. Here $\hat{L}=E^{2}-2 \cos \theta \cdot E+1$ with $E$ is the forwarding operator, i.e., $E x_{n}=x_{n+1}$. The lowest order solution may be written as

$$
x_{n}^{(0)}=A\left(n_{0}\right) \mathrm{e}^{i n \theta}+\text { c.c. }
$$

Here we have made it explicit that $A$ may be a function of $n_{0}$; its functional dependence is yet unknown and will be determined by the RG equation, which determination constitutes the central part of the RG method. The first order correction $x_{n}^{(1)}$ is given by

$$
x_{n}^{(1)}=-\frac{i}{2 \sin \theta}\left(n-n_{0}\right)\left(a_{1} A+3 a_{3}|A|^{2} A\right) \mathrm{e}^{i(n-1) \theta}+\text { c.c, }
$$

where we have omitted non-singular terms which are proportional to $\exp (i k n \theta)$ only with $k=0,2,3$. Notice that there appeared a secular term proportional to $n-n_{0}$. We remark that the solution of the first-order equation is not unique; one could add any term proportional to $x_{n}^{(0)}$. We have chosen the above form so that only new independent terms appear and the secular term vanishes at $n=n_{0}$, which assures the lowest order approximation is as good as 
possible. Up to $O\left(\epsilon^{2}\right)$,we have an approximate solution $x_{n}=x_{n}^{(0)}+\epsilon x_{n}^{(1)} \equiv x_{n}\left(\left(A\left(n_{0}\right) ; n_{0}\right)\right.$, which is only locally valid around $n=\forall n_{0}$; the validity as an approximate solution is lost due to the secular term as $\left|n-n_{0}\right|$ becomes large. It means that the naive perturbation expansion breaks, which is a well known fact.

One may say, however, that we have a family of discrete curves $x_{n}\left(A\left(n_{0}\right), n_{0}\right)$ with $n_{0}$ being a parameter characterizing the curves [8]. Each curve will become a good approximation for the exact solution around $n=n_{0}$ if $A\left(n_{0}\right)$ is suitably chosen. Therefore the "envelope" of the family of the curves may give a good approximation of the exact solution in a global domain. 12 The "envelope" of $x_{n}\left(\left(A\left(n_{0}\right) ; n_{0}\right)\right.$ is constructed as follows: We first impose that

$$
\Delta_{n_{0}} x_{n}\left(A\left(n_{0}\right) ; n_{0}\right)=0
$$

where $\Delta_{n_{0}}$ denotes the difference w.r.t. $n_{0}$. This is the basic equation of our method and we call it the RG/E equation. This is an equation to give $n_{0}$ and $A\left(n_{0}\right)$ as functions of $n_{0}(n)$. The "envelope" $x_{n}^{\mathrm{E}}$ is given by inserting this solution to $x_{n}\left(A\left(n_{0}\right) ; n_{0}\right) ; x_{n}^{\mathrm{E}}=$ $x_{n}\left(A\left(n_{0}(n)\right) ; n_{0}(n)\right)$. However, since we are constructing the "envelope" which contacts with the local solutions at $n=n_{0}$ so that the "envelope" give a good approximation, $n_{0}$ should be $n$,i.e., $n_{0}=n$ : Notice that this choice nicely eliminates the secular term from $x_{n}^{\mathrm{E}}$. Conversely speaking, $A(n)$ can be determined so that Eq.(4) gives $n_{0}=n$; this possibility is related with the "renormalizability" of the equation [7]. Thus we have

$$
\Delta A(n)=\epsilon \frac{\exp (-i \theta)}{2 i \sin \theta}\left(a_{1} A(n)+3 a_{3}|A(n)|^{2} A(n)\right),
$$

where $\Delta$ denotes the difference operator w.r.t. $n[13]$. This is the amplitude equation in a discrete form corresponding to Stuart-Landau equation [14 for continuum systems. $x_{n}^{\mathrm{E}}$ is thus given by

$$
x_{n}^{\mathrm{E}}=\left(A(n) e^{i n \theta}+\text { c.c }\right)+2 a_{2} \epsilon|A(n)|^{2}+\text { h.h.. }
$$

here h.h. denotes the higher harmonics. 
A significant point is that this function gives an approximate but uniformly valid solution in a global domain. Let us show this in a general setting. Let $\mathbf{X}_{n}={ }^{t}\left(X_{1 n}, X_{2 n}, \cdots, X_{d n}\right)$ and $\mathbf{F}\left(\mathbf{X}_{n}, n\right)={ }^{t}\left(F_{1}\left(\mathbf{X}_{n}, n\right), F_{2}\left(\mathbf{X}_{n}, n\right), \cdots, F_{d}\left(\mathbf{X}_{n}, n\right)\right)$; we assume that $\mathbf{F}\left(\mathbf{X}_{n}, n\right)$ is analytic with respect to $\mathbf{X}_{n}$. We consider the difference equation

$$
\Delta \mathbf{X}_{n}=\mathbf{F}\left(\mathbf{X}_{n}, n\right)
$$

We suppose that $\tilde{\mathbf{X}}_{n}\left(\mathbf{W}\left(n_{0}\right), n_{0}\right)$ is an approximate solution to the equation up to $O\left(\epsilon^{p}\right)$, where the $d$-dimensional vector $\mathbf{W}\left(n_{0}\right)$ denotes the initial values assigned at the initial time $n=n_{0}$. Here notice that $n_{0}$ is arbitrary. The envelope function is given by $\mathbf{X}_{n}^{E} \equiv \tilde{\mathbf{X}}_{n}(\mathbf{W}(n), n)$, where $\mathbf{W}(n)$ is the solution to the RG/E equation, $\Delta_{n_{0}} \tilde{\mathbf{X}}_{n}\left(\mathbf{W}\left(n_{0}\right), n_{0}\right)=O\left(\epsilon^{p}\right)$. Then one can show that $\mathbf{X}_{n}^{E}$ satisfies the original equation uniformly up to $O\left(\epsilon^{p}\right)$ as follows: $\Delta \mathbf{X}_{n}^{E}=\left.\Delta \tilde{\mathbf{X}}_{n}\left(\mathbf{W}\left(n_{0}\right), n_{0}\right)\right|_{n_{0}=n+1}+$ $\left.\Delta_{n_{0}} \tilde{\mathbf{X}}_{n}\left(\mathbf{W}\left(n_{0}\right), n_{0}\right)\right|_{n_{0}=n}=\mathbf{F}\left(\left.\tilde{\mathbf{X}}_{n}\left(\mathbf{W}\left(n_{0}, n_{0}\right), n\right)\right|_{n_{0}=n+1}+O\left(\epsilon^{p}\right)=\mathbf{F}\left(\tilde{\mathbf{X}}_{n}(\mathbf{W}(n), n), n\right)-\right.$ $\partial \mathbf{F} /\left.\partial \tilde{\mathbf{X}}_{n} \cdot \Delta_{n_{0}} \tilde{\mathbf{X}}_{n}\left(\mathbf{W}\left(n_{0}\right), n_{0}\right)\right|_{n_{0}=n}+O\left(\epsilon^{m \geq p}\right)=\mathbf{F}\left(\mathbf{X}_{n}^{E}, n\right)+O\left(\epsilon^{p}\right)$. Here we have utilized the $\mathrm{RG} / \mathrm{E}$ equation in the second and last equality, and the analyticity of $\mathbf{F}$ in the third equality. This concludes the proof.

We notice that the amplitude equation Eq.(5) is a first-order equation and a dynamical reduction is achieved in comparison with the original equation. In fact, in the polar representation $A(n)=R_{n} \exp \left(i \varphi_{n}\right), R_{n}$ and $\varphi_{n}$ satisfy the following equations,

$$
R_{n+1}=R_{n}-\epsilon / 2 \cdot\left(a_{1} R_{n}+3 a_{3} R_{n}^{3}\right)
$$

and $\varphi_{n+1}=\varphi_{n}-\epsilon / 2 \cdot \cot \theta\left(a_{1}+3 a_{3} R_{n}^{2}\right)$, respectively. Notice that $R_{n}$ is determined by the first order equation independently of $\varphi_{n}$, which in turn is given in terms of $R_{n}$. Thus one may say that the second-order dynamical system Eq.(1) is reduced to a first-order one. As is seen from the derivation, this reduced equation has a universal nature as has Stuart-Landau equation.

The first order equation for $R_{n}$ has simple qualitative properties depending on the signs and values of $a_{1}$ and $a_{3}$. For example, when $a_{1}<0$ but $a_{3}>0$, the equation is converted 
to $f_{n+1}=f_{n}+a f_{n}\left(1-f_{n}^{2}\right)$ with $f_{n}=\sqrt{3 a_{3} /\left|a_{1}\right|} R_{n}$ and $a \equiv \epsilon\left|a_{1}\right| / 2$. For $0<a<1$, the equation has a fixed point $f^{*}=1$, while for $1<a \leq 1.246$, the map shows a two-period behavior, and after that the map rapidly shows multiple-period behavior then eventually becomes chaotic.

As another example of ordinary difference equation, let us take the one which is derived as a discretization of the Rayleigh equation: $\ddot{x}+x=\epsilon \dot{x}\left(1-1 / 3 \cdot \dot{x}^{2}\right)$. We remark that the equation admit a limit cycle with the radius of 2 [15]. We take the following discretization; $\ddot{x} \rightarrow\left(x_{n+1}-2 x_{n}+x_{n-1}\right) / \Delta t^{2}, \dot{x} \rightarrow\left(x_{n}-x_{n-1}\right) / \Delta t$. That is, the central difference for the second derivative and the backward difference for the first derivative. Thus we have

$$
x_{n+1}-2 \cos \theta x_{n}+x_{n-1}=\bar{\epsilon}\left(x_{n}-x_{n-1}\right)\left(1-\frac{1}{3} \cdot \frac{\left(x_{n}-x_{n-1}\right)^{2}}{\Delta t^{2}}\right),
$$

where $\cos \theta=1-\Delta t^{2} / 2$ and $\bar{\epsilon}=\epsilon \Delta t$. We remark that this difference equation has a neutrally stable solution as the unperturbed one. This is not the case for other discretization schemes such as $\ddot{x} \rightarrow\left(x_{n+2}-2 x_{n+1}+x_{n}\right) / \Delta t^{2}$ and $\dot{x} \rightarrow\left(x_{n+1}-x_{n}\right) / \Delta t$. We put $1 / \Delta t=\omega$.

In the first-order approximation with respect to $\bar{\epsilon}$, we have

$$
\begin{aligned}
x_{n} & \equiv x_{n}\left(A\left(n_{0}\right) ; n_{0}\right)=\left[A\left(n_{0}\right)+\bar{\epsilon} \frac{\exp (-i \theta)}{2 i \sin \theta}\left(1-\omega^{2}\left|B\left(n_{0}\right)\right|^{2}\right) B\left(n_{0}\right) \cdot\left(n-n_{0}\right)\right] e^{i n \theta} \\
& -\frac{\bar{\epsilon}}{3} \omega^{2} \frac{B\left(n_{0}\right)^{3} \exp (3 i n \theta)}{(\exp (3 i \theta)-\exp (i \theta))(\exp (3 i \theta)-\exp (-i \theta))}+\text { c.c },
\end{aligned}
$$

where $B\left(n_{0}\right)=(\exp (i \theta)-1) A\left(n_{0}\right)$. Now the RG/E equation $\left.\Delta_{n_{0}} x_{n}\left(A\left(n_{0}\right) ; n_{0}\right)\right|_{n_{0}=n}=0$, gives the amplitude equation $\Delta A(n)=\bar{\epsilon} \exp (-i \theta) / 2 i \sin \theta \cdot\left(1-\omega^{2}|B(n)|^{2}\right) B(n)$, accordingly,

$$
A(n+1)=A(n)+z A(n)\left(1-|A(n)|^{2}\right)
$$

with $z \equiv \bar{\epsilon} \exp (-i \theta) / \cos \theta / 2$. If we take the polar representation $A(n)=R_{n} \exp \left(i \varphi_{n}\right)$, we have $R_{n+1}=R_{n}+\epsilon a^{\prime} R_{n}\left(1-R_{n}^{2}\right)$, and $\varphi_{n+1}=\varphi_{n}+\bar{\epsilon} \sin \theta / 2 \cdot\left(1-R_{n}^{2}\right)$ with $a^{\prime}=$ $\Delta t \cos \theta /(2 \cos \theta / 2)$. We note that $a^{\prime}$ as a function of $\Delta t$ is a parabola-like shape and takes the maximum about .6 at $\Delta t \simeq .9$; it vanishes at $\Delta t=0$ and $\Delta t \simeq 1.4$. Thus we see that $R_{n}$ goes up monotonically to a fixed point 1 .

With this $R_{n}$ and $\varphi_{n}$, the envelope $x_{n}^{\mathrm{E}}$ is given as 


$$
x_{n}^{\mathrm{E}} \equiv x_{n}(A(n) ; n)=2 R_{n} \cos \left(n \theta+\varphi_{n}\right)+\frac{\bar{\epsilon}}{12} \frac{\tan \theta}{\cos ^{3} \theta / 2} R_{n}^{3} \sin \left\{3\left(n \theta+\varphi_{n}\right)-\frac{3}{2} \theta\right\},
$$

which shows that the radius of the limit cycle is 2 , irrespective of the choice of $\Delta t$ in accordance with the original Rayleigh equation. We remark that it is not the case for other discretization schemes as given below Eq.(9).

Fig.1a shows $x_{n}^{E}$ given by Eq.(12) and the envelope $2 R_{n}$ together with the exact solution of Eq.(9) with $\epsilon=.4$ and $\Delta t=0.25$. One can see that the agreement is excellent in the global domain; notice that the results is obtained in the first order approximation. One can also see that the amplitude $2 R_{n}$ successfully describe the slow motion of the system. The characteristic features of the system as a dynamical system may be more clearly seen in Fig.1b where the behaviors in the "phase space" are shown. The agreement is excellent again. [16]

Finally, we consider partial difference equations. As an example, we take the difference equation which is given by a discretization of the 1-dimensional Swift-Hohenberg equation [17]; $\partial_{t} \phi(x, t)=\epsilon\left(\phi(x, t)-\phi(x, t)^{3}\right)-\left(1+\partial_{x}^{2}\right)^{2} \phi(x, t)$. With the discretization $\partial_{t} \phi \rightarrow(\phi(n, m+$ 1) $-\phi(n, m)) / \Delta t \equiv \Delta_{m} \phi(n, m) / \Delta t, \partial_{x}^{2} \phi \rightarrow(\phi(n+1, m)-2 \phi(n, m)+\phi(n-1, m)) / \Delta x^{2} \equiv$ $\Delta_{n}^{2} \phi(n, m) / \Delta x^{2}$, we have the following partial difference equation

$$
\hat{\mathcal{L}} \phi(n, m)=\epsilon\left(\phi(n, m)-\phi(n, m)^{3}\right)
$$

where $\hat{\mathcal{L}} \equiv \Delta_{m}+r\left(\Delta x^{2}+\Delta_{n}^{2}\right)^{2}$, with $r \equiv \Delta t / \Delta x^{4}$. We shall show that the difference equation admit a dynamical reduction giving an amplitude equation which is the analogue of the time-dependent Ginzburg-Landau equation in the continuum theory. The reason why the dynamical reduction is possible in the $R G$ method is that the difference equation is constructed so that the unperturbed equation has a neutrally stable solution.

Making the Taylor expansion $\phi=\phi_{0}+\epsilon \phi_{1}+\epsilon^{2} \phi_{2}+\cdots$, we have equations in the successive order $\hat{\mathcal{L}} \phi_{0}=0, \quad \hat{\mathcal{L}} \phi_{1}=\phi_{0}-\phi_{0}^{3}$, and so on. We consider an asymptotic solution at $m \rightarrow \infty$ and take the following neutrally stable solution as the 0-th order one;

$$
\phi_{0}(n, m)=A\left(n_{0}, m_{0}\right) e^{i n \theta_{x}}+\text { c.c }
$$


with $\theta_{x}=2 \operatorname{Sin}^{-1} \Delta x / 2$. Here we have made it explicit that the amplitude $A$ may depend in a yet unknown way on the initial time $m_{0}$ and point $n_{0}$. They will be determined by the $\mathrm{RG} / \mathrm{E}$ equation.

Then the first-order equation now reads

$$
\hat{\mathcal{L}} \phi_{1}=\left\{\left(A-3|A|^{2} A\right) e^{i n \theta_{x}}-A^{3} e^{3 i n \theta_{x}}\right\}+\text { c.c.. }
$$

A straightforward but somewhat tedious manipulation gives

$$
\begin{aligned}
\phi_{1}(n, m)= & {\left[\left[\mu_{1}\left(m-m_{0}\right)-\frac{\mu_{2} r^{-1}}{8 \sin ^{2} \theta_{x}}\left\{n^{(2)}-n_{0}^{(2)}+i e^{-i \theta_{x}}\left(n-n_{0}\right)\right\}\right]\right.} \\
& \left.\times\left(A-3|A|^{2} A\right) e^{i n \theta_{x}}-\frac{A^{3} e^{3 i(n+2) \theta_{x}}}{r\left(e^{3 i \theta_{x}}-e^{i \theta_{x}}\right)^{2}\left(e^{3 i \theta_{x}}-e^{-i \theta_{x}}\right)^{2}}\right]+ \text { c.c. },
\end{aligned}
$$

where $n^{(2)}=n(n-1)$ and $\mu_{1}+\mu_{2}=1$. Thus we have an approximate solution $\phi(n, m)=$ $\phi_{0}(n, m)+\epsilon \phi_{1}(n, m)$ which is valid only for $(n, m)$ around $n \sim n_{0}$ and $m \sim m_{0}$.

Now the RG/E equation $\left.\Delta_{m_{0}} \phi\right|_{m_{0}=m}=0$ and $\left.\Delta_{n_{0}} \phi\right|_{n_{0}=n}=0$ gives $\Delta_{m} A(n, m)=\epsilon \mu_{1}(1-$ $\left.3|A|^{2}\right) A$, and $\Delta_{n}^{2} A(n, m)=-\frac{\epsilon \mu_{2}}{4 r \sin ^{2} \theta_{x}}\left(1-3|A|^{2}\right) A$, respectively. Here we have utilized the fact $\Delta_{m} A=O(\epsilon)$ and $\Delta_{n} A=O(\epsilon)$, and neglected terms of $O\left(\epsilon^{2}\right)$. Thus noting that $\mu_{1}+\mu_{2}=1$, we reach the amplitude equation for the difference equation

$$
\Delta_{m} A(n, m)=4 r \sin ^{2} \theta_{x} \Delta_{n}^{2} A(n, m)+\epsilon\left(1-3|A(n, m)|^{2}\right) A(n, m)
$$

This is precisely the discretized form of the time-dependent Ginzburg-Landau equation: $\partial_{t} A(x, t)=4 \partial_{x}^{2} \phi(x, t)+\epsilon\left(1-3|A(x, t)|^{2}\right) A(x, t)$.

In summary, we have shown that the renormalization group method can be nicely extended to discrete systems, and that the method is useful as a tool for global asymptotic analysis and gives dynamical reduction of discrete systems. We have emphasized that the method is applicable for systems which have neutrally stable solution. It is to be remarked that this is also the case for equations which have unperturbed solutions on invariant stable and unstable manifolds. We have also given a notion of the discretization scheme that faithfully preserves the nature of the reduced dynamics irrespective of the magnitude of $\Delta t$. Finally, we notice that it is not trivial what discretization scheme preserves the integrability 
of differential equations which admit soliton solutions [18]. It will be interesting to examine if our discretization scheme based on the RG method can have a relevance to soliton theories.

We thank Prof. M. Yamaguti and Prof. R. Hirota for their interest in this work. This work is supported by the Grants-in-Aid of the Japanese Ministry of Education, Science and Culture, No. 07304065 and No. 08640396. 


\section{REFERENCES}

[1] P.Glandsdorff and I. Prigogine, Thermodynamics of Structure, Stability, and Fluctuations (John Wiley \& Sons, 1971); G. Nicolis and I. Prigogine, Self-Organization in Nonequilibrium Systems (John Wiley \& Sons, 1977); H. Haken, Synergetics, 3rd ed. (Springer, 1983); J.Guckenheimer and P. Holmes, Nonlinear Oscillations, Dynamical Systems, and Bifurcations and Vector Fields, 2nd ed. (Springer, 1986); M.C. Cross and P.C. Hohenberg, Rev. Mod. Phys. 65,851 (1993).

[2] Y. Kuramoto, Chemical Oscillations, Waves, and Turbulence (Springer, 1984).

[3] P. Manneville, Dissipative Structures and Weak Turbulence (Academic Press, 1990).

[4] G. Whitham, Linear and Nonlinear Waves, (Wiley, 1974).

[5] V. N. Bogaevski and A. Povzner, Algebraic Methods in Nonlinear Perturbation Theory, (Springer-Verlag, 1991); Y. Kuramoto, Prog. Theor. Phys. Suppl. 99, 244 (1989).

[6] E.C.G. Stuckelberg and A. Petermann, Helv. Phys. Acta 26, 499(1953); M. Gell-Mann and F. E. Low, Phys. Rev. 95, 1300 (1953).

[7] L. Y. Chen, N. Goldenfeld and Y. Oono, Phys. Rev. Lett.73,1311(1994); Phys. Rev.E 54, 376(1996); see also R. Graham, Phys. Rev. Lett. 76, 2185(1996); S. L. Woodruff, Studies in App. Mathematics 90, 225 (1993).

[8] T.Kunihiro, Prog.Theor.Phys. 94, 503(1995); 95, 835(1996)(E); Jpn. J. Ind. Appl. Math. 14, 51 (1997).

[9] S. Sasa, preprint patt-sol/9608008, to be published in Physica D.

[10] T. Kunihiro, Prog. Theor. Phys. 97, 179 (1997).

[11] The RG method works for systems which have a neutrally stable solution as the unperturbed solution. [10] Actually, the method is also applicable to equations which has unperturbed solutions lying on a stable and/or an unstable invariant manifold [1]. An 
example of such equations is $x_{n+2}-2 \cosh \theta x_{n+1}+x_{n}=\epsilon f\left(x_{n}, x_{n+1}\right)$.

[12] The envelope of a family of discrete curves may be defined as the curve which shares neighboring two points with each member of the family of curves.

[13] One can show that $\Delta A(n)=O(\epsilon)$, and we have neglected the terms of $O\left(\epsilon^{2}\right)$ in deriving Eq.(5).

[14] L. D. Landau and E. M. Lifscitz, Fluid Mechanics §27 (Pergamon, London, 1959); J. T. Stuart, J. Fluid Mech. 4,1 (1958).

[15] C.M. Bender and S.A. Orszag, Advanced Mathematical Methods for Scientists and Engineers (McGraw-Hill, 1978).

[16] We remark that the exact solution of Eq.(9) well reproduces the solution of the original differential equation even with $\Delta t$ as large as 0.25 .

[17] J. Swift and P.C. Hohenberg, Phys. Rev. A 15, 319 (1977).

[18] R. Hirota, J. Phys. Soc. Jpn. 43,1424, 2074, 2079(1977); ibid. 50, 3785 (1981); B. Grammaticos, A. Ramani and V. Papageorgiou, Phys. Rev. Lett. 67,1825 (1991). 


\section{Figure Captions}

Fig.1a The dots shows $x_{n}^{E}$ (Eq.(12)) v.s. $n \Delta t$ while the thin line the envelope $2 R_{n}$ for $\epsilon=.4$ and $\Delta t=0.25$. The bold line shows the exact solution of Eq.(9).

Fig.1b The behaviors in the "phase space"; the vertical axis denotes the "velocity" $v_{n} \equiv$ $\left(x_{n+1}-x_{n}\right) / \Delta t$, while the horizontal axis $x_{n}$. The dots shows our approximate solution whereas the solid line the exact one. 


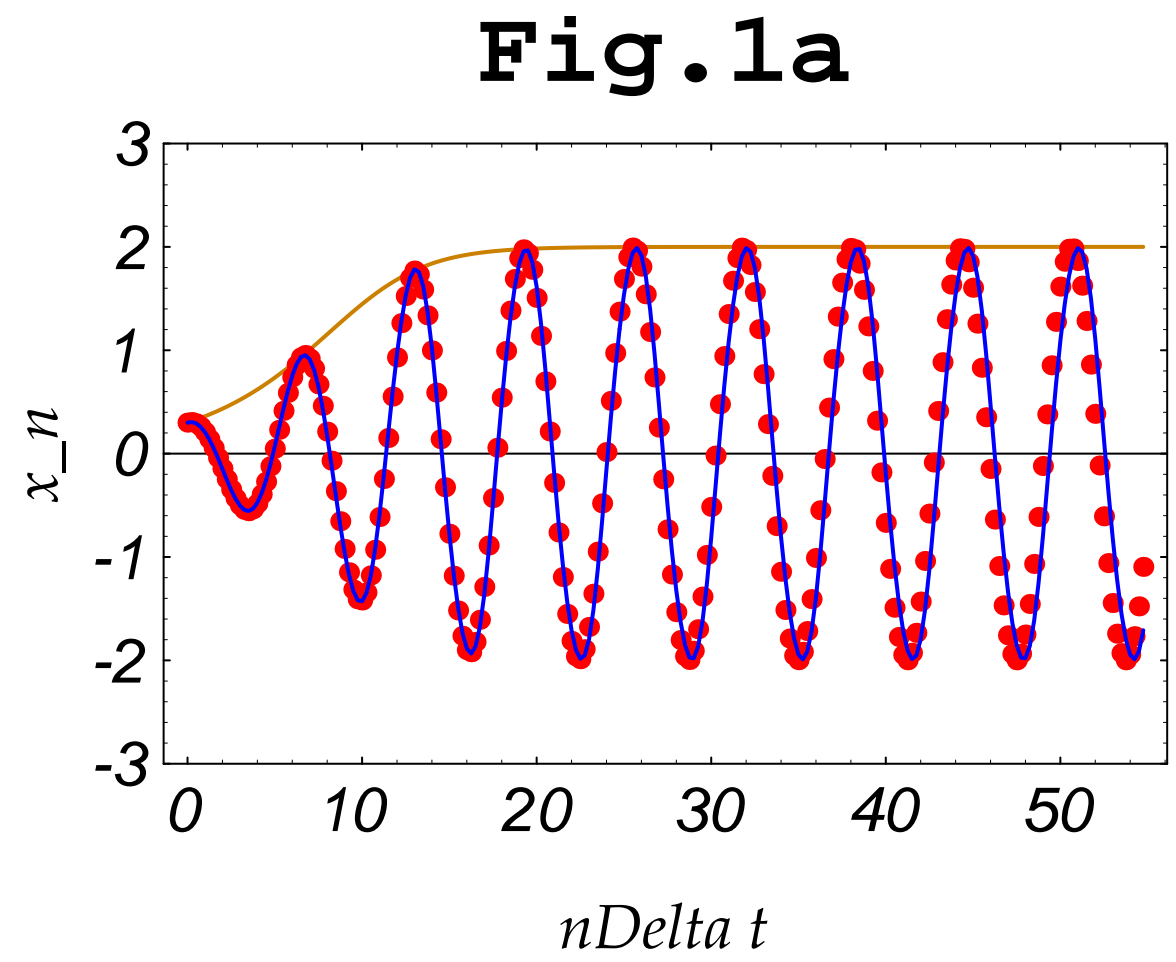

Fig. 1b

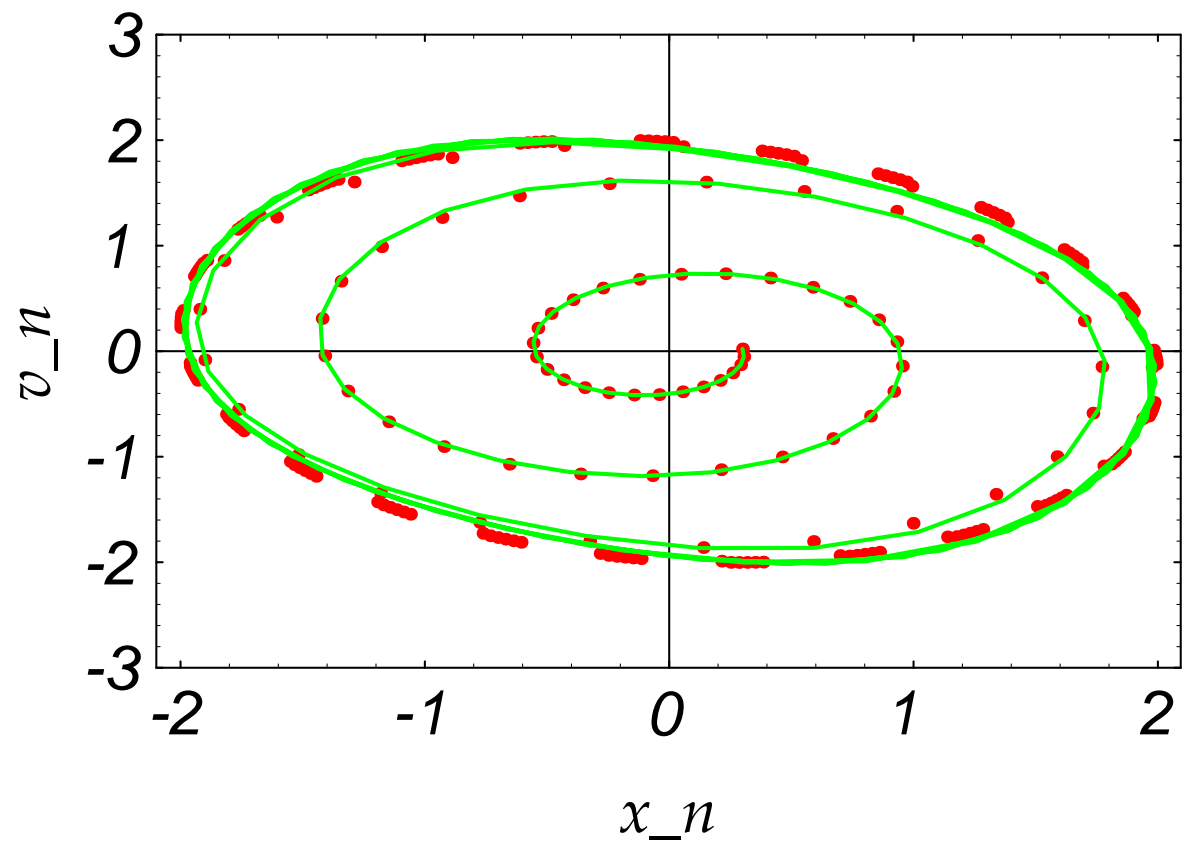

